\title{
A miniaturized drift tube ion mobility spectrometer for hand-held devices
}

\author{
André Ahrens, Stefan Zimmermann \\ Leibniz Universität Hannover, Institute of Electrical Engineering and Measurement Technology, \\ Department of Sensors and Measurement Technology, Appelstr. 9A, 30167 Hannover, Germany \\ e-mail: ahrens@geml.uni-hannover.de
}

\begin{abstract}
:
A miniaturized low-cost drift tube ion mobility spectrometer is presented. Due its small dimensions of about $15 \mathrm{~mm} \times 15 \mathrm{~mm} \times 90 \mathrm{~mm}$ it fits the needs of integration into hand-held devices. In this work, we show operation of a miniaturized drift tube design with a corona discharge ionization source. The major benefit of this ionization source is its non-radioactivity. Therefore, it is not restricted, in comparison to any radioactive sources, by any stringent regulations. By the need of a reaction region for such an ionization source, a three grid ion gate between the reaction and drift region is integrated. For demonstration, spectra of ammonia, acetone and dimethyl methylphosphonate (DMMP) are shown. The developed miniaturized low-cost ion mobility spectrometer has high resolving power of $\mathrm{R}_{\mathrm{P}}=60$ and detection limits in the $\mathrm{ppt}_{\mathrm{v}}$ range for measurement times within one second.
\end{abstract}

Key words: ion mobility spectrometer, hand-held, corona discharge

\section{Introduction}

Today, ion mobility spectrometers (IMS) are widely used in various analytical applications [1], especially in the field of safety and security [2]. Developing portable or hand-held devices for such applications requires miniaturization of the main components of the IMS. One major challenge is the development of a small drift tube with high analytical performance.

An approach to miniaturize a drift tube is simply downscaling of classic stacked designs [3]. However, manufacturing costs remain high [4]. Also micro structuring technologies for the manufacturing of miniaturized components are used [5]. Furthermore, miniaturized layouts using low temperature co-fired ceramics (LTCC) are known [6].

Besides miniaturization of the drift tube the ionization source should be also taken into account when thinking about the stringent regulations for radioactive ionization sources.

Therefore, in this work we show a miniaturized, low-cost IMS drift tube with a corona discharge ionization source.

\section{Design}

The drift tube electrodes are made of simple stainless steel straps soldered in shape of brackets on a common printed circuit board
(PCB). This PCB consists of alternating cooper straps and milled gaps. Together with the stainless steel straps closed electrodes are formed.

This electrode skeleton is covered by a housing made of polyether ether ketone (PEEK) and sealed with a two component epoxy adhesive.

Running an IMS with a corona discharge ionization sources also requires a reaction region. Therefore, a reaction region is designed similar to the drift tube, separated by an ion gate. The ion gate consists of a three grid setup shown in [7]. The functionality is shown in Fig. 1. Basically, in open state the second grid is on the mean potential between the first and third grid. In this state the ions pass the ion gate and enter the drift region. Switching the second grid to a higher potential, the second grid blocks the ions from entering the drift region, so that the gate is closed. The advantages and disadvantages of this ion gate will be discussed later.

All grids used in the IMS (counter electrode, ion gate and aperture grid) are made of stainless steel.

The ionization source housing, reaction region and drift region as well as the detector housing are sealed by $250 \mu \mathrm{m}$ foils of polytetrafluoroethylene (PTFE). 

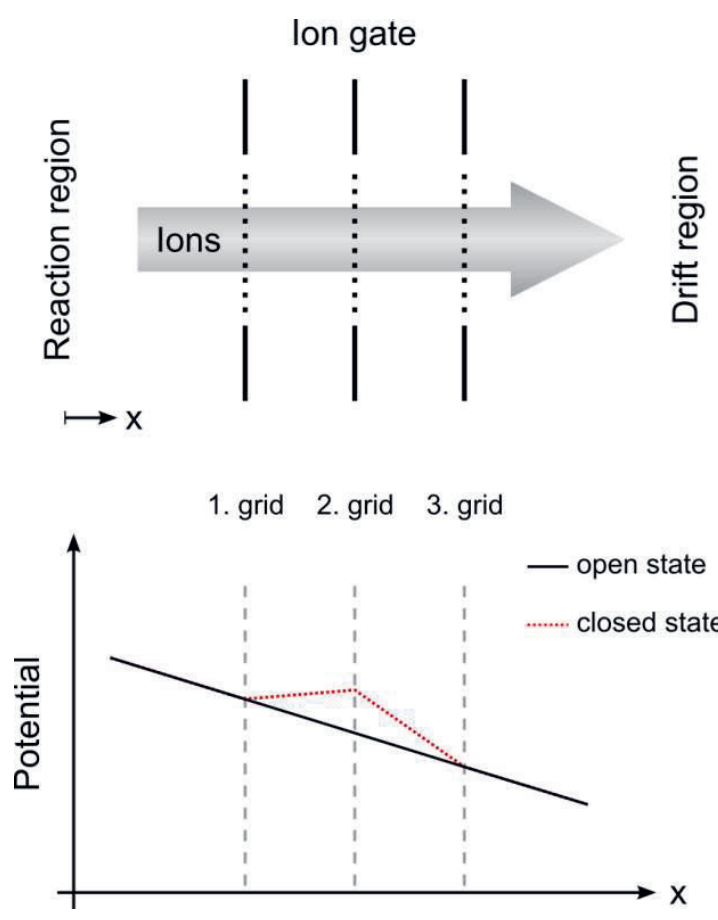

Fig. 1: Sketch of the used three grid ion gate (top) with its potentials for open and closed state (bottom).

The cannula of a medical syringe for neural therapy (Sterican by B.Braun) is used as a corona needle with $0.8 \mathrm{~mm}$ outer diameter (gauge 21).

Each drift electrode is connected to a resistive voltage divider giving a homogenous electrical field inside the reaction region and drift region. Passing both regions, the ions will be detected time resolved by a Faraday plate detector.

A sketch of the IMS drift tube with the corona discharge ionization source is shown in Fig. 2.

The overall length of the IMS, including the detector housing, the corona discharge ionization source and the reaction region is $90 \mathrm{~mm}$, with a cross-section of $15 \mathrm{~mm} \times 15 \mathrm{~mm}$.

A photo of this device is depicted in Fig. 3.

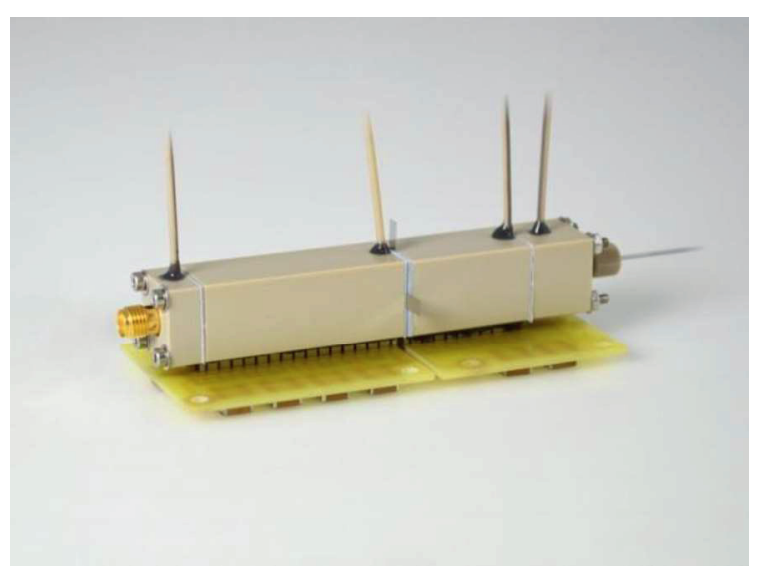

Fig. 3: Photo of the miniaturized low-cost IMS drift tube with corona ionization source.

\section{Experimental}

The IMS is driven with a drift gas flow of $80 \mathrm{mls} / \mathrm{min}$ of clean, dry air. Additionally, the sample gas flow is about $10 \mathrm{mls} / \mathrm{min}$ and carries the analytes. The electrical field inside the tube is generated by applying $1.2 \mathrm{kV}$ and $2.7 \mathrm{kV}$ to the resistive voltage dividers of the reaction region and drift region. A voltage of about of $3.4 \mathrm{kV}$ between the needle tip and the $2 \mathrm{~mm}$ distant counter electrode is applied to run a stable corona discharge.

The operating parameters of the IMS can be found in Tab. 1.

Dimethyl methylphosphonate (DMMP) (CAS 756-79-6), acetone (CAS 67-64-1) and ammonium carbonate (CAS 506-87-6) are used as analytes in this work. All substances are purchased by Sigma Aldrich.

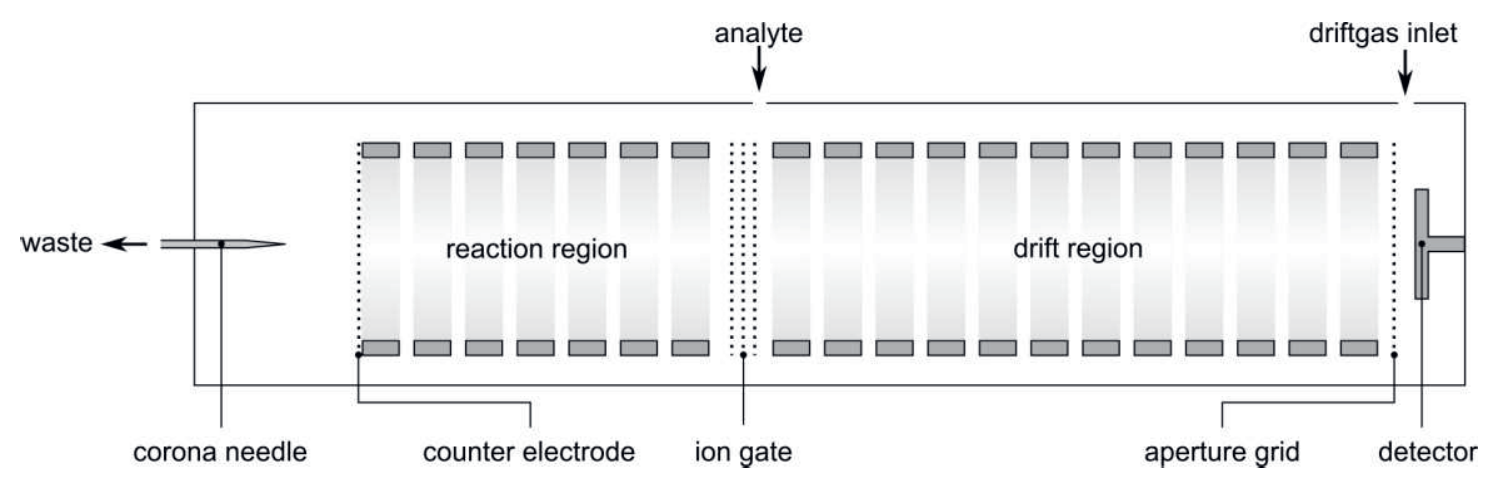

Fig. 2: Conceptual design of the IMS drift tube with corona ionization source. 
Tab. 1: Summarized operating parameters of the IMS.

\begin{tabular}{|c|c|}
\hline Length drift region & $40 \mathrm{~mm}$ \\
\hline Drift voltage & $2.7 \mathrm{kV}$ \\
\hline Length reaction region & $25 \mathrm{~mm}$ \\
\hline Reaction region voltage & $1.2 \mathrm{kV}$ \\
\hline lon gate block voltage & $120 \mathrm{~V}$ \\
\hline Needle-to-grid distance & $2 \mathrm{~mm}$ \\
\hline Needle-to-grid voltage & $3.4 \mathrm{kV}$ \\
\hline Drift gas flow & $80 \mathrm{mls} / \mathrm{min}$ \\
\hline Sample gas flow & $10 \mathrm{mls} / \mathrm{min}$ \\
\hline Temperature & $24^{\circ} \mathrm{C}$ \\
\hline IMS pressure & $1013 \mathrm{hPa}$ \\
\hline Dew Point of drift gas and \\
sample gas
\end{tabular}

\section{Results and Discussion}

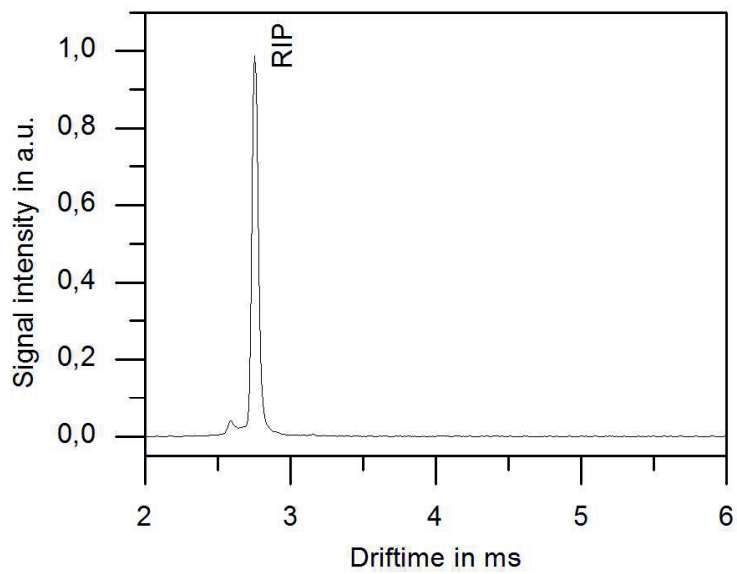

Fig. 4: Ion mobility spectrum of clean air.

Fig. 4 depicts an ion mobility spectrum of clean air without any analytes, so the reactant ion peak (RIP, $\left.\left[\mathrm{H}_{3} \mathrm{O}\right]^{+} \cdot\left(\mathrm{H}_{2} \mathrm{O}\right)_{n}\right)$ can be found at a drift time of $2.76 \mathrm{~ms}$. The resolving power of the IMS is about $R_{P}=60$. A smaller peak, detected at a drift time of $2.59 \mathrm{~ms}$ is not identified yet but probably another ion species formed by corona discharge ionization in clean air.

For demonstration, Fig. 5 shows the ion mobility spectrum of ammonia with a concentration of $39 \mathrm{ppb}_{\mathrm{v}}$ in air.

A calibration plot (peak amplitude over concentrations) for ammonia is given in Fig. 6 . Based on this plot, the limit of detection (LoD) for measurement times about $1 \mathrm{~s}$ is calculated using the $3 \sigma$ definition. Hence, ammonia can be detected down to a concentration of about $930 \mathrm{ppt}_{\mathrm{v}}$.

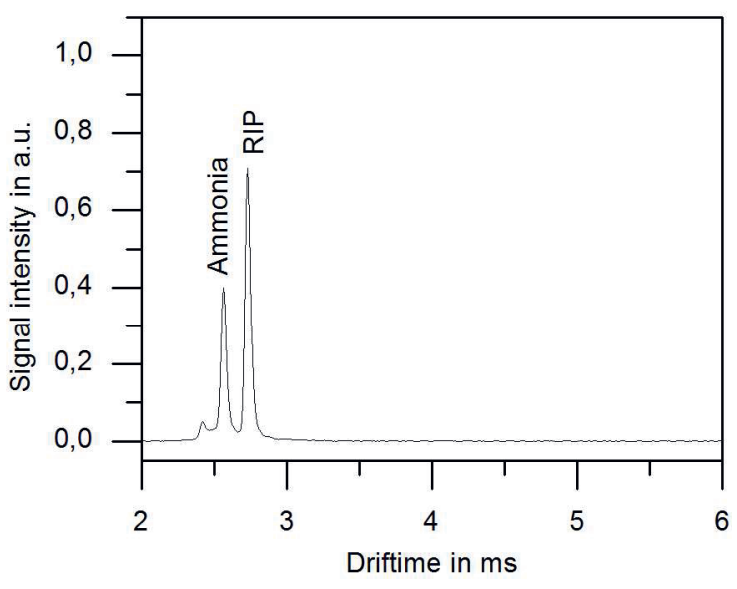

Fig. 5: Ion mobility spectrum of $39 p p b_{v}$ ammonia in clean air.

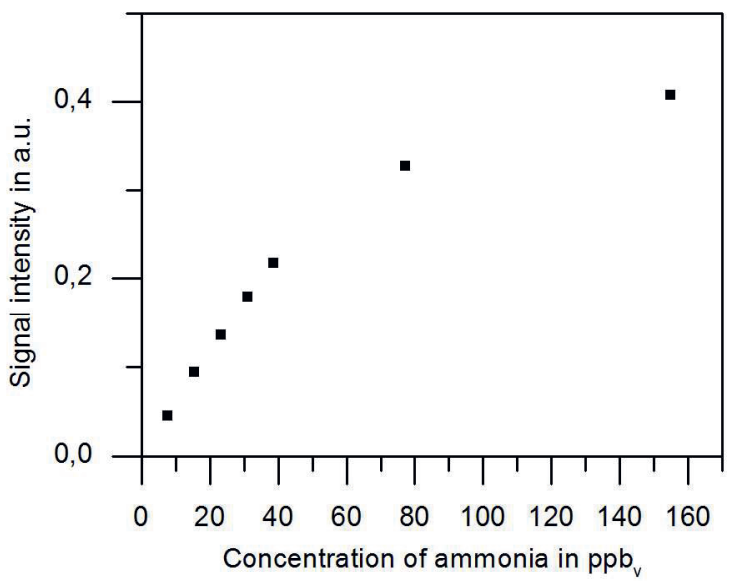

Fig. 6: Calibration plot of different concentrations of ammonia in clean air.

As another example, acetone is used. A concentration of $11 \mathrm{ppb}_{\mathrm{v}}$ of acetone results in the ion mobility spectrum depicted in Fig. 7. The acetone monomer peak can be detected at a drift time of $2.85 \mathrm{~ms}$, while the dimer peak is detected at $3.07 \mathrm{~ms}$. The LoD of acetone within a measurement time of $1 \mathrm{~s}$ is about $350 \mathrm{ppt}_{\mathrm{v}}$ for the monomer peak and $4.8 \mathrm{ppb}_{\mathrm{v}}$ for dimer peak.

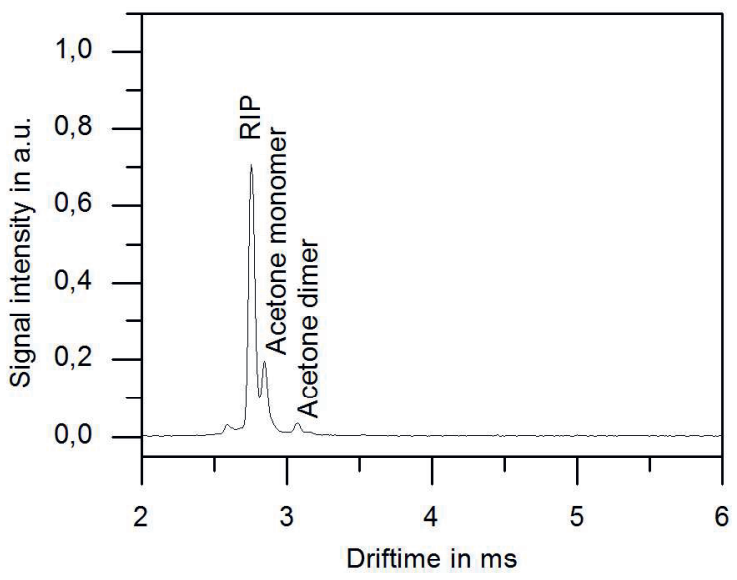

Fig. 7: Ion mobility spectrum of $11 p p b_{v}$ acetone in clean air. 
Finally, DMMP is measured as another model analyte. Therefore, the ion mobility spectrum of $24 \mathrm{ppb}_{\mathrm{v}}$ DMMP is shown in Fig. 8.

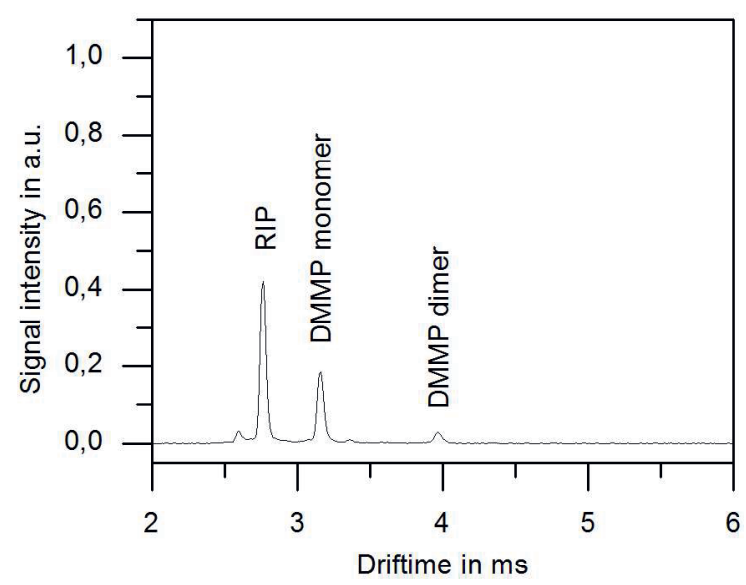

Fig. 8: Ion mobility spectrum of $24 p p b_{v}$ dimethyl methylphosphonate in clean air.

At a drift time of $3.16 \mathrm{~ms}$ a DMMP monomer peak is visible. Furthermore, at a drift time of $3.97 \mathrm{~ms}$ the DMMP dimer peak can be detected. While the LoD of DMMP monomer is about $560 \mathrm{ppt}_{\mathrm{v}}$ in the present setup, the LoD of the dimer is much higher at about $11.7 \mathrm{ppb}_{\mathrm{v}}$.

It can be recognized, that the LoDs of DMMP differs from the LoDs of other IMS setups. This might be caused by two different effects regarding the new design of the miniaturized low-cost drift tube.

At first, in the used experimental demonstrator the analyte is diluted by the drift gas due to the gas flows inside the IMS (see Fig. 2). This applies to all measurements and will be modified by a redesign of the gas flows in a newer version of the drift tube. Another issue is a discrimination effect of the ion gate for ions with lower ion mobility. In the operation mode, explained before, the ion gate opens for a fixed time, while the drift velocity of the ions passing the ion gate varies based on their ion mobility. Thus, ions with lower mobility are discriminated against the faster ions as they need longer time to pass the gate. This issue can be solved by changing the sequence of the grid potential as explained in [8].

\section{Conclusion}

In this work, we showed a miniaturized, lowcost drift tube design with a non-radioactive ionization source. Due its small dimensions of about $90 \mathrm{~mm}$ in length and just $15 \mathrm{~mm} \times 15 \mathrm{~mm}$ in cross-section it fits the needs of hand-held devices. Its analytical performance is demonstrated with ammonia, acetone and dimethyl methylphosphonate as model substances. The miniaturized low-cost IMS reaches high resolving power of about $R_{P}=60$. Furthermore, limits of detection in the mid ppt $_{v}$ range are determined for measurement times of $1 \mathrm{~s}$.

\section{Acknowledgement}

This project has received funding from the European Union's Horizon 2020 research and innovation programme under grant agreement No 653409.

\section{References}

[1] G.A. Eiceman, Z. Karpas, H.H. Hill, Ion mobility spectrometry. 3rd edn, CRC Press, Boca Raton, 2013.

[2] G.A. Eiceman, J.A. Stone, Anal. Chem. 76, 390A-397A (2004); doi: 10.1021/ac041665c

[3] J.S. Babis, R.P. Sperline, A.K. Knight, D.A. Jones, C.A. Gresham, M.B. Denton, Analytical and bioanalytical chemistry 395, 411-419 (2009); doi: 10.1007/s00216-009-2818-5.

[4] K.B. Pfeifer, R.C. Sanchez, Int. J. Ion Mobil. Spec. 5, 63-66 (2002).

[5] M. Teepe, J.I. Baumbach, A. Neyer, H. Schmidt, P. Pilzecker, IJIMS 2001, 60-64.

[6] K.B. Pfeifer, S.B. Rohde, K.A. Peterson, A.N. Rumpf, Int. J. Ion Mobil. Spec. 7 (2004).

[7] J. Langejuergen, M. Allers, J. Oermann, A. Kirk, S. Zimmermann, Anal. Chem. 86, 70237032 (2014); doi: 10.1021/ac5011662.

[8] S. Zimmermann, A.T. Kirk (Gottfried Wilhelm Leibniz Universität Hannover, Welfengarten 1, 30167 Hannover (DE)) WO2017021406A1, 2016. 